\title{
Combining Ability and Gene Action Studies in New Intra Hirsutum Hybrids with Suggested Breeding Strategies
}

\author{
V. Udaya ${ }^{* *}$ and Rajesh S. Patil ${ }^{2}$ \\ ${ }^{1}$ College of Agriculture, Dharwad, UAS Dharwad, India \\ ${ }^{2}$ Agricultural Research Station, Dharwad, UAS Dharwad, India \\ *Corresponding author
}

\section{A B S T R A C T}

The prime objective of any plant breeder is developing new cultivars with high yield. The present investigation aimed at estimating the general

\section{Keywords}

Combining ability, Gene effects,

Line, Tester

\section{Article Info}

Accepted:

10 March 2020

Available Online:

10 April 2020 combining ability among parents and specific combining ability in hybrids for various yield and related traits in upland cotton. Forty hybrids were obtained by crossing 8 elite lines with 5 selected testers in a Line $\times$ Tester fashion. All 40 hybrids, 13 parents along with 3 checks were evaluated under rainfed condition during kharif 2017-18at Agriculture Research Station, Dharwad Farm. Analysis of variance showed significant differences among parents and hybrids for most characters indicating presence of genetic variability. The ratio between variance due to GCA to that of SCA was less than unity for all characters indicating preponderance of non-additive gene effects in the inheritance of characters. Among lines, FLT-36, CPD-462 and among testers, SCS-1061, registered significant positive gca effects for seed cotton yield. Cross CPD-462 × SCS-1061 recorded high seed cotton vield with positive significant sca effects.

\section{Introduction}

Cotton (Gossypium spp.) is a crop of prosperity having influence on man and matter and is called the 'King of fibre'. It is also rightly called as 'White Gold'. Cotton is one of the most important commercial crops and forms the back bone of Indian textile industry. India has the largest area under cotton (122.38 lakh ha) and is one of the largest producers (361.00 lakh bales) with productivity of501 $\mathrm{kg} \mathrm{ha}^{-1}$ (Anon., 2019).

Cotton breeding mainly focus on development of cultivars with early maturity, high seed cotton yield, combined with good fibre properties. To combine all these traits there is a need to identify suitable genotypes to be 
used in crossing programs to generate populations. Combining ability aids in the elucidation of nature and magnitude of gene action leading to selection of parents. The concept of combining ability was introduced by Sprague and Tatum (1942). General combining ability ( $g c a$ ) is average performance of a parent in a series of crosses and specific combining ability (sca) designates those cases in which certain combinations perform relatively better or worse than would be expected on the basis of average performance of lines involved. Though the per se performance of parents is very important, selection on the basis of combining ability effects is a more robust procedure.

Kempthorne (1957) introduced the Line $x$ Tester analysis method which provides valuable information about general and specific combining ability variances, their effects thus helping in identification of good general combiners and specific promising cross combinations (Muthuswamy et al., 2003).

\section{Materials and Methods}

The genetic material for present investigation consisted of 40 hybrids which were obtained by crossing the eight elite linesviz.,FLT-36, FLT-44, FLT-31, FLT-28, SG-1, SG-2, EL-4, CPD-462 with the five testers viz., NNDC-30, NNDC-24, NNDC-59, IH-11 and SCS-1061 in $\mathrm{L} \times \mathrm{T}$ fashion along with three standard checks viz., DHH-11, DHH-263 and Ajeet199 Bt. All the above genotypes were never used in any hybridisation activity till now. Additionally, the genotypes were carefully chosen to represent diversity in plant type features also of which two traits, plant height and sympodial length, were dealt with in the present study. This investigation was carried out in Agricultural Research Station,
Dharwad Farm during kharif 2017. The genetic material was laid out in a randomized block design with three replications. The spacing of $90 \mathrm{~cm}$ between the rows and $60 \mathrm{~cm}$ between plants within row for hybrids and 90 $\mathrm{cm}$ between the rows and $20 \mathrm{~cm}$ between plants for parents was followed. The hybrids and parents were randomised amongst themselves and were sown in separate but adjacent plots. Each entry was sown in two rows of 4.80 meters length. The crop was raised under rainfed condition. All agronomic management practices according to recommended package of practices were followed to raise a good crop. Suitable plant protection measures were carried out to control pests and diseases at appropriate time.

\section{Results and Discussion}

The analysis of variance (Table 1) revealed that there were significant differences among the genotypes for all thirteen characters investigated. The mean sum of squares for parents was significant for most of the characters except for number of seeds per boll, ginning outturn, lint index, sympodial length at 50 per cent plant height and SPAD values. The lines showed significant differences for most traits except for number of seeds per boll, ginning outturn, lint index and sympodial length at 50 per cent plant height and SPAD values.

Among the testers, significant differences were observed for number of monopodia, seed cotton yield, number of seeds per boll and ginning outturn. The interaction between lines and testers was significant for plant height, number of monopodia per plant, number of sympodia per plant and number of bolls per plant. The interaction between parents and hybrids was found to be significant for all traits except for SPAD values. 
Table.1 Analysis of variance for yield and yield component characters in cotton (G. hirsutum L.)

\begin{tabular}{|c|c|c|c|c|c|c|c|c|c|c|c|c|c|c|}
\hline $\begin{array}{l}\text { Source of } \\
\text { variation }\end{array}$ & df & DFF & PH & NMP & NSP & SL@50\% & NBP & BW & NSP & SI & GOT & LI & SPAD & SCY \\
\hline Replication & 2 & 0.23 & 12.61 & 0.06 & 0.86 & 20.42 & 2.10 & 0.36 & 4.92 & 0.54 & 2.39 & 0.83 & 1.51 & 20500.67 \\
\hline Treatments & 52 & $4.20 * *$ & $428.11 * *$ & $0.54 * *$ & $4.30 * *$ & $65.01 * *$ & $40.73 * *$ & $0.43 * *$ & $7.93 * *$ & $1.30^{* *}$ & $10.01 * *$ & $1.06 * *$ & $4.70 * *$ & $190320.30 * *$ \\
\hline Parents & 12 & $4.07 *$ & $474.18 * *$ & $0.43 * *$ & $8.31 * *$ & 35.83 & $6.35 * *$ & $0.31 * *$ & 6.41 & $0.97 * *$ & 8.74 & 0.46 & 4.80 & $61995.08 * *$ \\
\hline Lines & 7 & $5.22 * *$ & $472.28 * *$ & $0.45 * *$ & $11.05^{* *}$ & 28.63 & $3.83 *$ & $0.41 * *$ & 1.89 & $1.10^{*}$ & 2.34 & 0.10 & 5.03 & $59678.24 *$ \\
\hline Testers & 4 & 1.99 & 95.36 & $0.44 * *$ & 0.59 & 45.60 & 3.11 & 0.13 & $12.66 * *$ & 0.86 & $19.73 * *$ & 0.87 & 5.36 & $76341.59 *$ \\
\hline $\begin{array}{l}\text { Lines vs. } \\
\text { Testers }\end{array}$ & 1 & 4.31 & $2002.82 * *$ & $0.31 * *$ & $19.94 * *$ & 47.18 & $36.92 * *$ & 0.29 & 13.10 & 0.46 & 9.62 & 1.28 & 0.95 & 20826.92 \\
\hline $\begin{array}{l}\text { Parents vs. } \\
\text { Crosses }\end{array}$ & 1 & $17.18 * *$ & $4151.20 * *$ & $7.34 * *$ & $60.64 * *$ & $1073.87 * *$ & $1430.07 * *$ & $4.48 * *$ & $96.96 * *$ & $7.45^{* *}$ & $120.04 * *$ & $19.45^{* *}$ & 0.49 & $701120.00 * *$ \\
\hline Crosses & 39 & $3.91 * *$ & $318.47 * *$ & $0.40^{* *}$ & 1.62 & $48.12 *$ & $15.69 * *$ & $0.36 * *$ & $6.11 *$ & $1.25^{* *}$ & 7.57 & $0.78 *$ & $4.78 *$ & $165425.50 * *$ \\
\hline Error & 104 & 1.75 & 78.25 & 0.03 & 1.68 & 29.20 & 1.62 & 0.13 & 3.60 & 0.40 & 5.26 & 0.48 & 2.68 & 23666.29 \\
\hline
\end{tabular}

Table.2 Analysis of variance for combining ability

\begin{tabular}{|c|c|c|c|c|c|c|c|c|c|c|c|c|c|}
\hline Genetic Parameter & DFF & PH & NMP & NSP & SL@50\% & NBP & BW & NSP & SI & GOT & LI & SPAD & SCY \\
\hline$\sigma^{2}$ GCA & 0.02 & 8.80 & 0.01 & 0.016 & 2.04 & 1.30 & 0.01 & 0.11 & 0.05 & 0.19 & 0.01 & 0.23 & 110.94 \\
\hline$\sigma^{2} \mathrm{SCA}$ & 1.16 & 79.87 & 0.11 & 0.019 & 5.72 & 2.34 & 0.06 & 0.48 & 0.20 & 1.02 & 0.16 & 0.25 & 44242.05 \\
\hline$\sigma^{2} \mathrm{GCA} / \sigma^{2} \mathrm{SCA}$ & 0.018 & 0.110 & 0.144 & 0.853 & 0.358 & 0.556 & 0.160 & 0.231 & 0.265 & 0.185 & 0.045 & 0.929 & 0.003 \\
\hline Contribution (\%) of Lines & 9.22 & 24.56 & 25.68 & 23.56 & 24.53 & 55.72 & 23.69 & 27.19 & 33.49 & 31.54 & 12.12 & 40.69 & 27.37 \\
\hline Contribution $(\%)$ of Testers & 3.55 & 2.44 & 4.70 & 9.169 & 13.39 & 3.80 & 6.78 & 5.97 & 5.60 & 2.50 & 7.01 & 7.82 & 3.55 \\
\hline Contribution $(\%)$ of Line $\times$ Tester & 87.21 & 79.92 & 69.31 & 67.26 & 62.06 & 40.47 & 69.51 & 68.83 & 60.89 & 65.94 & 80.86 & 51.48 & 69.06 \\
\hline
\end{tabular}

where, $\mathrm{DFF}=$ days to 50 per cent flowering, $\mathrm{PH}=$ plant height $(\mathrm{cm}), \mathrm{NMP}=$ number of monopodia per plant, $\mathrm{NSP}=$ number of sympodia per plant, $\mathrm{SL} @ 50 \%=$ sympodial length at 50 per cent plant height $(\mathrm{cm}), \mathrm{NBP}=$ number of bolls per plant, $\mathrm{BW}=$ boll weight $(\mathrm{g}), \mathrm{NSP}=$ number of seeds per plant, $\mathrm{SI}=$ seed index $(\mathrm{g})$, GOT= ginning outturn (g), LI= lint index (g), SPAD = Soil Plant Analysis Development meter values, $\mathrm{SCY}=$ Seed cotton yield $(\mathrm{kg} / \mathrm{ha})$ 
Table.3 General combining ability of parents in Line $\times$ Tester analysis in cotton (G. hirsutum L.)

\begin{tabular}{|c|c|c|c|c|c|c|c|c|}
\hline Parents & $\begin{array}{c}\text { Days to } \\
50 \% \\
\text { flowering }\end{array}$ & $\begin{array}{l}\text { Plant height } \\
\quad(\mathrm{cm})\end{array}$ & $\begin{array}{l}\text { Number of } \\
\text { monopodia } \\
\text { per plant }\end{array}$ & $\begin{array}{l}\text { Number of } \\
\text { sympodia } \\
\text { per plant }\end{array}$ & $\begin{array}{c}\text { Sympodial } \\
\text { length at } \\
50 \% \text { plant } \\
\text { height }(\mathrm{cm})\end{array}$ & $\begin{array}{c}\text { Number of } \\
\text { bolls per } \\
\text { plant }\end{array}$ & $\begin{array}{c}\text { Boll weight } \\
\text { (g) }\end{array}$ & $\begin{array}{c}\text { Number of } \\
\text { seeds per boll }\end{array}$ \\
\hline \multicolumn{9}{|l|}{ Lines } \\
\hline FLT-36 & $-0.67^{*}$ & -3.34 & $0.20 * *$ & 0.42 & 1.76 & $3.79 * *$ & 0.18 & -0.73 \\
\hline FLT-44 & -0.01 & $-6.58 * *$ & $-0.16 * *$ & 0.51 & 1.20 & 0.34 & -0.19 & -0.63 \\
\hline FLT-31 & 0.19 & -1.24 & $0.20 * *$ & -0.00 & -0.78 & 0.06 & $0.23 *$ & $-1.16^{*}$ \\
\hline FLT-28 & 0.49 & 3.23 & 0.093 & 0.08 & $2.99 *$ & $-0.73 *$ & $-0.22 *$ & 0.22 \\
\hline SG-1 & 0.13 & 0.39 & 0.00 & -0.47 & -0.86 & $-2.42 * *$ & -0.17 & 0.70 \\
\hline SG-2 & 0.27 & $11.36 * *$ & $-0.33 * *$ & 0.20 & 1.04 & $-1.29 * *$ & -0.01 & $1.09 *$ \\
\hline EL-4 & -0.29 & -2.25 & $-0.14 * *$ & -0.38 & -2.23 & 0.38 & 0.09 & 0.52 \\
\hline CPD-462 & -0.12 & -1.56 & $0.14^{* *}$ & -0.36 & $-3.12 *$ & -0.14 & 0.08 & -0.03 \\
\hline S.Ed & 0.29 & 2.36 & 0.04 & 0.441 & 1.27 & 0.34 & 0.09 & 0.53 \\
\hline CD at $5 \%$ & 0.57 & 4.71 & 0.09 & 0.87 & 2.54 & 0.68 & 0.19 & 1.05 \\
\hline \multicolumn{9}{|l|}{ Testers } \\
\hline NNDC-30 & 0.02 & 0.28 & -0.03 & 0.01 & $2.06^{*}$ & $0.65^{*}$ & 0.02 & -0.13 \\
\hline NNDC-24 & -0.13 & 0.31 & $-0.08 *$ & 0.32 & 1.25 & 0.23 & $0.15^{*}$ & -0.03 \\
\hline NNDC-59 & -0.22 & 1.48 & 0.03 & 0.03 & -0.40 & -0.49 & -0.02 & -0.44 \\
\hline IH-11 & 0.39 & -3.05 & $0.13^{* *}$ & -0.37 & -1.81 & 0.08 & -0.11 & -0.01 \\
\hline SCS-1061 & -0.06 & 0.97 & -0.04 & 0.01 & -1.10 & -0.48 & -0.04 & 0.61 \\
\hline S.Ed & 0.22 & 1.87 & 0.03 & 0.34 & 1.00 & 0.27 & 0.07 & 0.42 \\
\hline CD at $5 \%$ & 0.45 & 3.72 & 0.07 & 0.69 & 2.00 & 0.54 & 0.15 & 0.83 \\
\hline
\end{tabular}


Table 3. Contd.....

\begin{tabular}{|c|c|c|c|c|c|c|c|c|}
\hline Parents & $\begin{array}{c}\text { Seed index } \\
\text { (g) }\end{array}$ & $\begin{array}{c}\text { Ginning } \\
\text { outturn } \\
(\%)\end{array}$ & $\begin{array}{c}\text { Lint index } \\
\text { (g) }\end{array}$ & $\begin{array}{l}\text { SPAD meter } \\
\text { value }\end{array}$ & $\begin{array}{l}\text { Seed cotton } \\
\text { yield }(\mathrm{kg} / \mathrm{ha})\end{array}$ & UHML (mm) & $\begin{array}{l}\text { Fibre strength } \\
\quad(g / t e x)\end{array}$ & $\begin{array}{c}\text { Micronaire } \\
\text { ( } \mu \mathrm{g} / \text { /inch })\end{array}$ \\
\hline \multicolumn{9}{|l|}{ Lines } \\
\hline FLT-36 & -0.29 & 0.61 & -0.02 & $1.25 * *$ & $248.63 * *$ & -0.64 & 0.11 & 0.05 \\
\hline FLT-44 & $-0.34^{*}$ & $1.54 * *$ & 0.20 & 0.35 & -76.17 & 0.07 & 0.53 & 0.01 \\
\hline FLT-31 & 0.13 & 0.08 & 0.12 & 0.46 & $-109.50^{*}$ & 0.69 & 1.25 & 0.05 \\
\hline FLT-28 & -0.08 & -0.50 & -0.19 & -0.14 & $-90.30 *$ & 0.69 & 0.33 & -0.14 \\
\hline SG-1 & 0.01 & $-1.20^{*}$ & $-0.32 *$ & $-1.01^{*}$ & -68.88 & -0.70 & -1.42 & -0.09 \\
\hline SG-2 & $0.89 * *$ & $-1.22 *$ & 0.20 & $-1.32 * *$ & -48.50 & -0.02 & -1.06 & 0.16 \\
\hline EL-4 & -0.16 & 0.38 & 0.01 & 0.56 & -6.43 & 0.13 & 0.21 & -0.03 \\
\hline CPD-462 & -0.16 & 0.30 & -0.02 & -0.14 & $151.16^{* *}$ & -0.20 & 0.03 & -0.01 \\
\hline S.Ed & 0.17 & 0.50 & 0.16 & 0.40 & 41.95 & & & \\
\hline CD at $5 \%$ & 0.34 & 1.01 & 0.31 & 0.80 & 83.53 & & & \\
\hline \multicolumn{9}{|l|}{ Testers } \\
\hline NNDC-30 & -0.07 & -0.09 & -0.08 & -0.39 & -16.55 & -0.10 & -0.35 & -0.08 \\
\hline NNDC-24 & -0.14 & -0.22 & -0.16 & -0.01 & 34.03 & -0.16 & -0.55 & 0.08 \\
\hline NNDC-59 & 0.01 & -0.24 & -0.04 & -0.37 & -49.52 & 0.09 & 0.18 & 0.01 \\
\hline IH-11 & $0.28 *$ & 0.16 & 0.22 & 0.32 & -34.78 & 0.01 & 0.37 & -0.07 \\
\hline SCS-1061 & -0.07 & 0.39 & 0.06 & 0.45 & $66.83^{*}$ & 0.16 & 0.35 & 0.06 \\
\hline S.Ed & 0.13 & 0.40 & 0.12 & 0.32 & 33.17 & & & \\
\hline CD at $5 \%$ & 0.27 & 0.80 & 0.25 & 0.63 & 66.04 & & & \\
\hline
\end{tabular}

*, ** significant at 5 and 1 per cent respectively 
Table.4 Specific combining ability effects of intra hirsutum hybrids for yield and yield component characters

\begin{tabular}{|c|c|c|c|c|c|c|c|c|}
\hline Cross & $\begin{array}{c}\text { Days to } \\
\mathbf{5 0 \%} \\
\text { flowering }\end{array}$ & $\begin{array}{l}\text { Plant height } \\
\text { (cm) }\end{array}$ & $\begin{array}{c}\text { Number of } \\
\text { monopodia } \\
\text { per plant }\end{array}$ & $\begin{array}{l}\text { Number of } \\
\text { sympodia } \\
\text { per plant }\end{array}$ & $\begin{array}{c}\text { Sympodial } \\
\text { length at } \\
50 \% \text { plant } \\
\text { height }(\mathrm{cm})\end{array}$ & $\begin{array}{l}\text { Boll weight } \\
\text { (g) }\end{array}$ & $\begin{array}{l}\text { Number of } \\
\text { bolls per } \\
\text { plant }\end{array}$ & $\begin{array}{c}\text { Number of } \\
\text { seeds per boll }\end{array}$ \\
\hline FLT-36 $\times$ NNDC-30 & $-2.50 * *$ & 4.73 & 0.11 & 0.14 & 3.15 & 0.40 & $-2.47 * *$ & 0.54 \\
\hline FLT-36 $\times$ NNDC-24 & $1.69 *$ & 5.49 & $-0.36 * *$ & 0.30 & 3.29 & -0.27 & 1.07 & -0.48 \\
\hline FLT-36 $\times$ NNDC-59 & 1.01 & -9.60 & $0.50 * *$ & -0.07 & -1.03 & -0.22 & $2.24 * *$ & 0.49 \\
\hline FLT-36 $\times$ IH-11 & -0.67 & -4.53 & -0.18 & 0.53 & -2.63 & 0.13 & -0.31 & 1.74 \\
\hline FLT-36 $\times$ SCS-1061 & 0.47 & 3.90 & -0.07 & -0.91 & -2.78 & -0.04 & -0.53 & -2.30 \\
\hline FLT-44 $\times$ NNDC-30 & -0.68 & 2.77 & -0.19 & 0.05 & 0.60 & 0.05 & -0.29 & 0.41 \\
\hline FLT-44 $\times$ NNDC-24 & 0.17 & $-11.62 *$ & 0.06 & -1.25 & $-5.75^{*}$ & -0.01 & 0.43 & -0.18 \\
\hline FLT-44 $\times$ NNDC-59 & 0.60 & $-12.22 *$ & 0.13 & 0.63 & -4.74 & -0.35 & $1.71 *$ & 0.09 \\
\hline FLT-44 × IH-11 & 0.46 & 9.31 & 0.17 & -0.16 & 4.01 & 0.11 & $-1.86^{*}$ & 0.74 \\
\hline FLT-44 × SCS-1061 & -0.56 & $11.75^{*}$ & -0.17 & 0.72 & $5.88 *$ & 0.20 & 0.00 & -1.06 \\
\hline FLT-31 $\times$ NNDC-30 & $1.59 *$ & 1.97 & $0.51 * *$ & -0.16 & -0.02 & -0.06 & $1.82 *$ & -0.58 \\
\hline FLT-31 $\times$ NNDC-24 & $-1.69 *$ & 6.26 & $0.43 * *$ & 0.73 & 4.52 & 0.09 & -0.68 & -0.68 \\
\hline FLT-31 $\times$ NNDC-59 & 0.29 & 2.70 & $-0.35 * *$ & -1.24 & -1.85 & 0.41 & 1.01 & -0.31 \\
\hline FLT-31 × IH-11 & -0.36 & -9.22 & $-0.65 * *$ & 0.62 & -1.73 & -0.32 & -0.88 & 0.98 \\
\hline FLT-31 × SCS-1061 & 0.16 & -1.71 & 0.06 & 0.04 & -0.93 & -0.11 & -1.27 & 0.59 \\
\hline FLT-28 $\times$ NNDC-30 & -1.07 & $-22.17 * *$ & -0.17 & 0.14 & -0.86 & -0.14 & $3.24 * *$ & -2.14 \\
\hline FLT-28 $\times$ NNDC-24 & -0.14 & 8.38 & -0.19 & 0.03 & 3.17 & 0.33 & -0.86 & 1.35 \\
\hline FLT-28 $\times$ NNDC-59 & $1.98 * *$ & 6.89 & -0.11 & -0.33 & 1.73 & 0.00 & -1.29 & 0.06 \\
\hline FLT-28 $\times$ IH-11 & -0.25 & 1.89 & $0.78 * *$ & 0.46 & -3.12 & -0.10 & -0.16 & 0.92 \\
\hline FLT-28 $\times$ SCS-1061 & -0.50 & 5.00 & $-0.29 * *$ & -0.31 & -0.92 & -0.07 & -0.92 & -0.19 \\
\hline SG-1 $\times$ NNDC-30 & $1.79 * *$ & $14.59 * *$ & -0.08 & 0.90 & -2.67 & $-0.49 *$ & $-1.84 *$ & -1.15 \\
\hline SG-1 $\times$ NNDC-24 & -0.12 & 0.09 & 0.10 & -0.86 & 0.13 & 0.20 & $1.84^{*}$ & 0.40 \\
\hline SG-1 $\times$ NNDC-59 & $-1.80 * *$ & $-10.60 *$ & -0.02 & -0.24 & -0.54 & -0.31 & -1.42 & 0.85 \\
\hline SG-1 $\times$ IH-11 & -1.12 & -6.80 & 0.04 & -0.24 & 1.87 & -0.21 & 0.83 & -0.89 \\
\hline SG-1 $\times$ SCS-1061 & 1.25 & 2.70 & -0.03 & 0.44 & 1.21 & $0.81 * *$ & 0.59 & 0.79 \\
\hline SG-2 $\times$ NNDC-30 & 0.47 & -7.57 & $-0.41 * *$ & -1.17 & 0.08 & 0.05 & 0.43 & 1.08 \\
\hline
\end{tabular}




\begin{tabular}{|c|c|c|c|c|c|c|c|c|}
\hline SG-2 $\times$ NNDC-24 & 0.10 & -6.21 & $0.63 * *$ & 0.71 & $-5.88^{*}$ & -0.07 & $1.79 *$ & -0.51 \\
\hline SG-2 $\times$ NNDC-59 & -0.16 & 3.29 & $-0.22 *$ & 0.61 & $6.77 *$ & -0.26 & 0.20 & -1.50 \\
\hline SG-2 $\times$ IH-11 & 0.54 & $10.75^{*}$ & $-0.25^{*}$ & 0.07 & -2.27 & $0.51 *$ & -0.16 & -1.44 \\
\hline SG-2 × SCS-1061 & -0.96 & -0.26 & $0.26^{*}$ & -0.23 & 1.30 & -0.23 & $-2.26 * *$ & $2.37 *$ \\
\hline EL-4 $\times$ NNDC-30 & 1.01 & 8.57 & $0.33 * *$ & 0.01 & 3.25 & -0.22 & -0.30 & $2.68^{*}$ \\
\hline EL-4 × NNDC-24 & 0.79 & -5.32 & $-0.48 * *$ & 0.57 & 1.45 & -0.15 & $-2.24 * *$ & 0.58 \\
\hline EL-4 $\times$ NNDC-59 & -1.18 & -2.35 & 0.05 & -0.53 & -4.84 & $0.51 *$ & -1.16 & -0.17 \\
\hline EL-4 × IH-11 & -0.79 & 3.90 & -0.10 & -0.53 & 3.51 & 0.19 & $1.59 *$ & -1.21 \\
\hline EL-4 × SCS-1061 & 0.17 & -4.79 & 0.20 & 0.48 & -3.36 & -0.31 & $2.12 * *$ & -1.89 \\
\hline CPD-462 × NNDC-30 & -0.60 & -2.90 & -0.09 & 0.06 & -3.52 & 0.41 & -0.58 & -0.85 \\
\hline CPD- $462 \times$ NNDC- 24 & -0.82 & 2.92 & -0.18 & -0.24 & -0.93 & -0.10 & -1.34 & -0.48 \\
\hline CPD-462 $\times$ NNDC-59 & -0.73 & $21.89 * *$ & 0.02 & 1.18 & 4.50 & 0.23 & -1.30 & 0.49 \\
\hline CPD-462 × IH-11 & $2.20 * *$ & -5.30 & 0.19 & -0.75 & 0.36 & -0.30 & 0.95 & -0.85 \\
\hline CPD-462 × SCS-1061 & -0.04 & $-16.59 * *$ & 0.04 & -0.25 & -0.41 & -0.23 & $2.28 * *$ & 1.69 \\
\hline S.Ed & 0.64 & 5.29 & 0.10 & 0.69 & 2.85 & 0.22 & 0.77 & 1.18 \\
\hline CD at $5 \%$ & 1.29 & 10.54 & 0.21 & 1.38 & 5.68 & 0.44 & 1.54 & 2.36 \\
\hline
\end{tabular}

Table.4 Contd.....

\begin{tabular}{|c|c|c|c|c|c|c|c|c|}
\hline Cross & $\begin{array}{l}\text { Seed index } \\
\text { (g) }\end{array}$ & $\begin{array}{c}\text { Ginning } \\
\text { outturn }(\%)\end{array}$ & $\begin{array}{c}\text { Lint index } \\
\text { (g) }\end{array}$ & $\begin{array}{l}\text { SPAD meter } \\
\text { value }\end{array}$ & $\begin{array}{c}\text { Seed cotton } \\
\text { yield }(\mathrm{kg} / \mathrm{ha})\end{array}$ & $\begin{array}{c}\text { UHML } \\
\text { (mm) }\end{array}$ & $\begin{array}{l}\text { Fibre strength } \\
\quad(g / t e x)\end{array}$ & $\begin{array}{c}\text { Micronaire } \\
(\mu \mathrm{g} / \text { inch })\end{array}$ \\
\hline FLT-36 $\times$ NNDC-30 & 0.25 & -1.04 & -0.12 & -1.05 & -15.44 & 0.72 & -0.57 & 0.06 \\
\hline FLT-36 $\times$ NNDC-24 & $-1.07 * *$ & 0.77 & -0.48 & 0.83 & -45.53 & -0.60 & -0.69 & 0.46 \\
\hline FLT-36 $\times$ NNDC-59 & 0.34 & 0.06 & 0.21 & 0.07 & 46.85 & 1.28 & 0.90 & -0.06 \\
\hline FLT-36 $\times$ IH-11 & 0.18 & -1.52 & -0.32 & 1.20 & -3.38 & -1.32 & -3.09 & -0.31 \\
\hline FLT-36 $\times$ SCS-1061 & 0.31 & 1.73 & 0.71 & -1.05 & 17.49 & 0.18 & 0.18 & 0.14 \\
\hline FLT-44 ×NNDC-30 & 0.41 & 0.29 & 0.35 & 0.85 & -70.14 & -0.30 & 0.02 & -0.17 \\
\hline FLT-44 $\times$ NNDC-24 & 0.42 & 0.96 & 0.55 & 1.24 & 122.93 & 0.14 & 2.54 & -0.43 \\
\hline FLT-44 $\times$ NNDC-59 & -0.57 & 1.03 & -0.10 & -0.73 & 145.32 & -0.12 & 0.72 & 0.31 \\
\hline FLT-44 $\times$ IH-11 & 0.18 & -1.22 & -0.22 & $-1.93 *$ & -120.58 & -2.12 & -0.67 & -0.07 \\
\hline FLT-44 ×SCS-1061 & -0.44 & -1.08 & -0.59 & 0.58 & -77.53 & 1.57 & 1.92 & -0.17 \\
\hline FLT-31 $\times$ NNDC-30 & 0.05 & 1.15 & 0.34 & -1.14 & -126.81 & -0.06 & 0.20 & 0.02 \\
\hline
\end{tabular}




\begin{tabular}{|c|c|c|c|c|c|c|c|c|}
\hline FLT-31 $\times$ NNDC-24 & 0.32 & -0.16 & 0.12 & 0.85 & -16.89 & 0.75 & 1.82 & -0.02 \\
\hline FLT-31 $\times$ NNDC-59 & -0.26 & $-2.58 *$ & $-0.86^{*}$ & -0.68 & $376.65^{* *}$ & 1.05 & -1.83 & -0.05 \\
\hline FLT-31 × IH-11 & -0.43 & 1.46 & 0.17 & 0.24 & 88.58 & 1.27 & 2.72 & -0.11 \\
\hline FLT-31 × SCS-1061 & 0.32 & 0.13 & 0.24 & 0.73 & $-321.53 * *$ & -2.00 & -4.77 & 0.27 \\
\hline FLT-28 $\times$ NNDC-30 & $-0.93^{*}$ & $2.47 *$ & 0.10 & 0.97 & $301.32 * *$ & -0.46 & 0.62 & 0.14 \\
\hline FLT-28 $\times$ NNDC-24 & 0.47 & -1.92 & -0.22 & -1.71 & -20.93 & 0.92 & 1.40 & -0.21 \\
\hline FLT-28 $\times$ NNDC-59 & 0.61 & 0.02 & 0.39 & -0.22 & -152.87 & -1.40 & -1.72 & 0.20 \\
\hline FLT-28 $\times$ IH-11 & -0.07 & -0.91 & -0.30 & 0.62 & -109.44 & -0.72 & -0.74 & 0.27 \\
\hline FLT-28 $\times$ SCS-1061 & -0.07 & 0.34 & 0.04 & 0.35 & -18.07 & 0.48 & 0.18 & -0.18 \\
\hline SG-1 $\times$ NNDC-30 & 0.26 & 0.20 & 0.15 & 0.15 & -163.60 & 0.18 & 1.34 & 0.00 \\
\hline SG-1 $\times$ NNDC-24 & 0.11 & 1.08 & 0.37 & -0.60 & $239.51 *$ & -0.10 & -0.62 & 0.16 \\
\hline SG-1 $\times$ NNDC-59 & -0.42 & -1.81 & -0.68 & 0.68 & -142.04 & 1.24 & 1.10 & -0.11 \\
\hline SG-1 × IH-11 & -0.52 & 0.49 & -0.19 & 0.59 & 118.96 & -0.62 & -0.92 & -0.14 \\
\hline SG-1 $\times$ SCS-1061 & 0.57 & 0.01 & 0.35 & -0.82 & -52.82 & 0.51 & 1.01 & 0.08 \\
\hline SG-2 $\times$ NNDC-30 & -0.46 & -0.10 & -0.32 & -0.11 & 99.35 & 0.19 & 0.19 & -0.30 \\
\hline SG-2 $\times$ NNDC-24 & $-0.82 *$ & 0.79 & -0.26 & -0.24 & 144.10 & -1.03 & -0.83 & 0.22 \\
\hline SG-2×NNDC-59 & -0.07 & 0.20 & -0.04 & 0.94 & $-206.17 *$ & -0.13 & 0.89 & 0.15 \\
\hline SG-2 $\times$ IH-11 & $1.47 * *$ & 1.44 & $1.33 * *$ & 0.55 & 56.08 & -1.03 & -0.85 & -0.04 \\
\hline SG-2 $\times$ SCS-1061 & -0.12 & $-2.34 *$ & -0.71 & -1.14 & -93.37 & -1.51 & -2.51 & 0.15 \\
\hline EL-4 $\times$ NNDC-30 & -0.18 & $-2.79 *$ & $-0.83^{*}$ & 0.82 & -180.21 & 0.93 & 0.71 & 0.07 \\
\hline EL-4 $\times$ NNDC-24 & 0.11 & -0.90 & -0.18 & -1.52 & -127.96 & 2.07 & 1.39 & -0.32 \\
\hline EL-4 $\times$ NNDC-59 & 0.53 & 1.02 & 0.61 & 0.81 & 164.75 & -0.04 & -1.18 & 0.14 \\
\hline EL-4 $\times$ IH-11 & -0.54 & 1.42 & 0.02 & -0.16 & $248.68 * *$ & 0.24 & 0.30 & -0.18 \\
\hline EL-4 $\times$ SCS-1061 & 0.07 & 1.25 & 0.39 & 0.06 & -105.27 & 0.52 & 0.48 & -0.44 \\
\hline CPD-462 $\times$ NNDC -30 & 0.61 & -0.18 & 0.33 & -0.49 & 155.52 & 0.22 & 0.20 & 0.36 \\
\hline CPD-462 ×NNDC-24 & 0.47 & -0.64 & 0.10 & 1.15 & $-295.23 * *$ & -0.38 & 1.16 & -0.06 \\
\hline CPD-462 $\times$ NNDC-59 & -0.16 & 2.04 & 0.48 & -0.87 & $-232.50 *$ & 0.64 & 0.40 & -0.03 \\
\hline CPD-462 ×IH-11 & -0.28 & -1.17 & -0.49 & -1.10 & $-278.91 * *$ & -0.32 & 0.42 & 0.20 \\
\hline CPD-462 × SCS-1061 & -0.64 & -0.05 & -0.42 & 1.30 & $651.12 * *$ & -0.88 & -1.80 & 0.01 \\
\hline S.Ed & 0.38 & 1.14 & 0.36 & 0.91 & 93.82 & & & \\
\hline CD at $5 \%$ & 0.77 & 2.27 & 0.71 & 1.81 & 186.79 & & & \\
\hline
\end{tabular}

$*, * *$ significant at 5 and 1 per cent respectively 
The mean sum of squares with respect to hybrids was found to be significant for all traits except for number of sympodia per plant and ginning outturn. This depicted the presence of considerable genetic difference among the hybrids.

The results of ANOVA for combining ability are given in Table 2. The ratio between GCA variance to SCA variance was less than unity for all the traits investigated indicating predominance of non-additive gene action for all yield and its component traits. These results were in accordance with the earlier findings of Deshpande et al., (2008), Basal et al., (2011), Alkuddsi et al., (2013)and Monicashree et al., (2017).

The combining ability study revealed that among lines, FLT-36 was a good general combiner for number of bolls per plant and seed cotton yield, FLT-44was a good general combiner for ginning outturn and a negative general combiner for number of monopodia per plant and FLT-31 was a good general combiner for boll weight.

Among testers, NNDC-30 was a good general combiner for sympodial length at 50 per cent plant height and number of bolls per plant, NNDC-24 was a good general combiner for boll weight and SCS-1061 was a good general combinerfor seed cotton yield (Table 3). These parents can be utilized in crop improvement programs via multiple or strategic crossing schemes to develop high yielding varieties and also varieties that could fit in to high density planting. Less plant height, and sympodial length with fewer or no monopodia are traits which help in breeding compact genotypes to suit high density planting.

Among crosses,CPD-462 $\times$ SCS-1061, FLT$28 \times$ NNDC-30, EL-4 $\times$ IH-11 had high sca effects for seed cotton yield, number of bolls per plant and ginning outturn (Table 4). Crosses which exhibited positive sca effects for different traits could be exploited in heterosis breeding.

Finally, the present study showed that combining ability of parents and hybrids is important in deciding the breeding strategy. Superior lines FLT-36, FLT-44, FLT-31, EL1, CPD-462 and testers NNDC-30, IH-11 and SCS-1061 with high gca effects for yield and its components can be used in generating populations to extract new varieties. Also, ideotype considerations to breed genotypes for specific growing conditions have also been pointed out.

\section{References}

Alkuddsi, Y., Patil, S. S., Manjula, S. M., Patil, B. C., Nadaf, H. L. and Nandihalli, B. S., 2013, Development of heterotic groups ( $G$. hirsutum vs $G$. barbadense) based on combining ability and inter specific hybrids performance for yield and fiber quality traits. Cotton Genomics and Genetics, 4(3): 33-44.

Anonymous, 2019, Annu. Rep. (2018-19), All India Co-ordinated Cotton Improvement Project, 2018-19, p. A1-A10.

Basal, H., Canavar, O., Khan, N. U. and Cerit, C. S., 2011, Combining ability and heterotic studies through line $\times$ tester in local and exotic upland cotton genotypes, Pak. J. Bot., 43(3): 16991706.

Deshpande, N.P., Dheva, N.G., Potdukhe, N.R., Parmar, J.N. and Wadodkar, M.B., 2008, Combining ability study for yield and morphological traits in interspecific crosses between $G$. hirsutum L. and G. barbadense L. Intl. J. Plant Sci., 3(1): 158-162.

Kempthrone, O., 1957, An Introduction to Genetic Statistics, New York, John Wiley and Sons, pp. 456-471. 
Monicashree, C., Amala, P. B. and Gunasekaran, M., 2017, Heterosis studies for yield and fibre quality traits in upland cotton (Gossypium hirsutum L.). Int. J. Pure App. Biosci., 5 (3): 169-186.

Muthuswamy, A., Vivekanandan, P. and Jayaramachandran, M., 2003,
Combining ability and gene action for fibre characters in upland cotton (Gossypium hirsutum L.). J. Indian Soc. Cotton Improv., 28(2): 127-131.

Sprague, G. F. and Tatum, L. A., 1942, General vs. specific combining ability in a single cross corn. J. Amer. Soc. Agron., 34: 923-932.

\section{How to cite this article:}

Udaya, V, and Rajesh S. Patil. 2020. Combining Ability and Gene Action Studies in New Intra Hirsutum Hybrids with Suggested Breeding Strategies. Int.J.Curr.Microbiol.App.Sci. 9(04): 1227-1236. doi: https://doi.org/10.20546/ijcmas.2020.904.145 